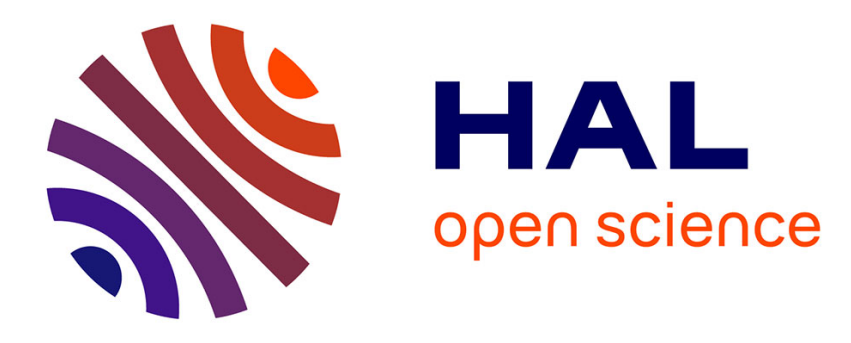

\title{
Electrostatically actuated thermal switch device for caloric film
}

\author{
Morgan Almanza, Lucas Depreux, Fabien Parrain, Martino Lobue
}

\section{To cite this version:}

Morgan Almanza, Lucas Depreux, Fabien Parrain, Martino Lobue. Electrostatically actuated thermal switch device for caloric film. Applied Physics Letters, 2018, 112 (8), pp.083901. 10.1063/1.5009618 . hal-01706363

\section{HAL Id: hal-01706363 https://hal.science/hal-01706363}

Submitted on 11 Feb 2018

HAL is a multi-disciplinary open access archive for the deposit and dissemination of scientific research documents, whether they are published or not. The documents may come from teaching and research institutions in France or abroad, or from public or private research centers.
L'archive ouverte pluridisciplinaire HAL, est destinée au dépôt et à la diffusion de documents scientifiques de niveau recherche, publiés ou non, émanant des établissements d'enseignement et de recherche français ou étrangers, des laboratoires publics ou privés. 


\title{
Electrostatically actuated thermal switch device for caloric film
}

\author{
Morgan Almanza, ${ }^{1}$ Lucas Depreux, ${ }^{1}$ Fabien Parrain, ${ }^{2}$ and Martino LoBue ${ }^{1}$ \\ 1) SATIE, CNRS, ENS Paris-Saclay, Université Paris-Saclay, 94235 Cachan, \\ France \\ 2) C2N, Université Paris-Sud, CNRS, 91405 Orsay, France
}

An innovative thermal switch device using a thin metallic film electrostatically actuated by an electrode mainly conceived for caloric cooling is studied. Our study focuses on the characterization of the thermal conductance at the interface for the "on" and "off" states. Our set-up uses the current passing through the metallization of the film as a heater while the temperature is deduced from the measurement of its electrical resistivity. Using a thermal diffusion model and our measurements, we deduce the on and off states thermal conductances and we achieve an on/off conductance ratio of $10^{3}$. Lastly, we use a simple finite-time thermodynamic model to estimate the efficiency at maximum power we would obtain by integrating a standard electrocaloric film in our thermal switch. The result is a micro-refrigerator working at $85 \%$ of Carnot efficiency with a power density of $228 \mathrm{~W} \mathrm{~g}^{-1}$ which is far more than what it have been currently demonstrated.

Keywords: heat exchange, electrostatic actuator, thermal switch, caloric material, electrocaloric

A thermal switch (TS) is a device able to change its effective thermal conductance in order to control the rate of heat transfer across a junction. Development of efficient thermal switches is a key issue in the field of solid state cooling where the refrigerant substance (e.g. a caloric material $^{1}$ ) is also used as a heat exchanger by alternately switching its thermal contact between the hot and cold reservoirs $^{2}$ as schematically depicted in Fig. 1.
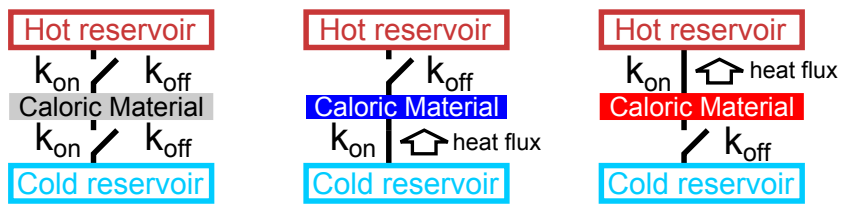

FIG. 1. Example of a single stage cooling system based on caloric material (electrocaloric material ECM) with two thermal switches and two heat reservoirs (heat storage).

The efficiency and the power density of the cooling system ${ }^{2,3}$ are strictly related to the performances of the thermal switch. These performances depend on the following characteristics : (1) high ratio between thermal conductances $k_{o n}$ and $k_{o f f}$ (respectively the high and low conductance states), $\eta=k_{o n} / k_{o f f}$, (2) high $k_{o n}$ value, (3) small switching time (i.e. time to switch between on and off states and vice versa). The key parameters to characterize the device are $k_{o n}$ and $k_{o f f}$. Even before discussing in detail each of these characteristics, it is apparent that an efficient TS will show: (4) low power to switch between states and low heat dissipation, (5) low heat capacity of the TS as a whole.

Throughout the past decades, researchers have tried to design TSs based on different approaches, such as acting on the molecular orientation in liquid crystal to change thermal conductivity ${ }^{4}$, or using solid state thermal rectification ${ }^{5,6}$ reporting respectively a conductance ratio of 3.3 and until 1.4. Such low values could hardly respond to the needs of an actual device. In solid state a number of strategies are still exploited ${ }^{7}$. We also point out that thermoelectrics have no interest to use caloric effect to achieve cooling because the major problem limiting the thermoelectric cooling (i.e a low factor of merit) is the same problem that limit the effect of the thermal switch. The principle of the thermosyphon, improved by the coalescence effect ${ }^{8}$ reached a conductance ratio $\eta=10^{2}$. The displacement of droplets to open or short-circuit a thermal path ${ }^{9}$ achieved a conductance ratio $\eta=24$. These two solutions present a higher $\eta$ than the previous ones, yet they add elements that inevitably increase the overall thermal capacity and may increase the switching time. A promising solution to tackle the five points previously defined is to use small mechanical displacements and/or mechanical pressure, to change the thermal contact. In this case, the mechanical interface has to be well-controlled, using for instance a liquid-metal micro-droplet array ${ }^{10}$ to maintain a proper thermal contact in spite of the motion. In this case, the measured conductance ratio is around 8 using Si contact, or 24 using a liquid-metal micro-droplet array on the Silicon. A "sliding" silicon heat switch ${ }^{11}$ has been conceived; the switch is composed of two symmetric parts both made of alternating stripes of Silicon (high conductivity material) and of lower conductivity materials. Sliding the two parts on top of each other changes the conductance: a ratio up to 59 has been reported. However, in both cases, the liquid-metal/sliding parts approaches might be problematic, due to viscosity/friction.

In order to achieve efficient thermal switching, the surface-to-thickness ratio has to be as high as possible. Thin films are therefore excellent candidates for mechanical heat switches as long as we can handle them, due to their fragility. Here, to achieve repeatable switching with thin films, we use electrostatic forces for actuation. The concept has been used and it is shown that it is possible to establish reversible and repeatable mechanical contact led by electrostatic forces with a thin gold film ${ }^{12}$. This method has recently demonstrated its effectiveness for an electrocaloric based cooling system ${ }^{13}$ where a power density of $3 \mathrm{~W} \mathrm{~g}^{-1}$ and a coefficient of performance of 13 have been reported. Prediction of conductance ratio as high 
as $10^{6}$ let foresee the opportunity of a real breakthrough for caloric cooling ${ }^{12}$.

Unfortunately, data on conductance ratios are sparse at best ${ }^{4,10}$ with reported values ranging from 3.3 to 59 . The gap between theoretical values and measurements shows how complex thermal conductivity measurements at interfaces can be, and proves the need for more reliable characterization techniques.

In this letter, we present a set-up to measure the thermal conductance of a thermal switch led by an electrostatic field acting on a thin metallic film as shown in Fig. 2. First, we describe the set-up in detail. Then, we explain how measurements and simulations are combined to calculate the thermal conductance in terms of an equivalent air gap. Finally, based on our results, we discuss the potential impact of the integration of a caloric material in our switch on solid state cooling techniques. We report a conductance ratio of $10^{3}$, which is the highest directly measured value so far.

A device based on a single cooling stage consists in a caloric material placed between two thermal switches as shown in Fig. 1. In this letter, we characterize just one thermal switch, the dash dot line in Fig. 1 refers to the axis of symmetry of the complete cooling device. Our set-up is composed of several layers, as shown in Fig. 2.

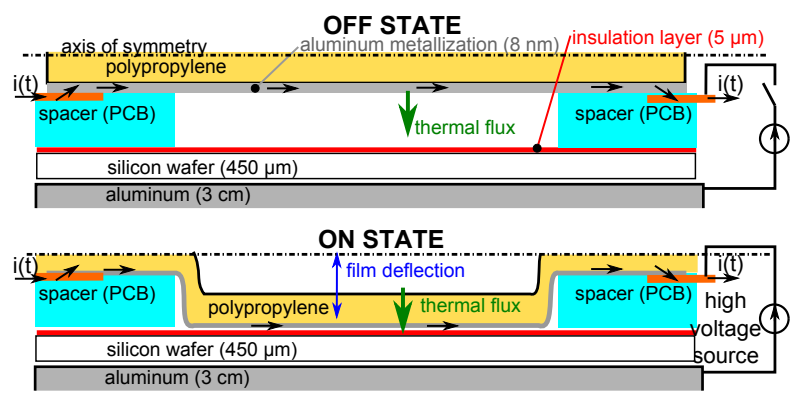

FIG. 2. Simplified side view of our experiment from top to bottom: a Polypropylene film $(8 \mu \mathrm{m})$ metallized with Aluminum on the bottom $(\sim 8 \mathrm{~nm})$, an epoxy with copper $(500 \mu \mathrm{m})$ used as a spacer, and a polished Silicon wafer $(\sim 450 \mu \mathrm{m})$ (surface roughness $<1 \mathrm{~nm})$ with an added insulation layer (a $5 \mu \mathrm{m}$ spin-coated Polyimide film), on which the film collapses.

In the cooling device, the metallized Polypropylene film will be the caloric material. By applying an electrical tension between the electrodes (i.e. to the metallization on the bottom of the film and the Silicon), we induce a deflection of the film causing it to collapse on the dielectric, which is on the wafer; this displacement and the resulting contact change constitute the switching mechanism. The film is clamped to the spacer so that at rest, the stress maintains the film suspended (i.e. in the "off" state-as shown in Fig. 3 and Fig. 4), when the voltage is applied the film collapses on the insulation layer due to electrostatic force. The "on" state is then reached when the film has fully collapsed on the insulation layer.

The insulation layer is made of Polyimide

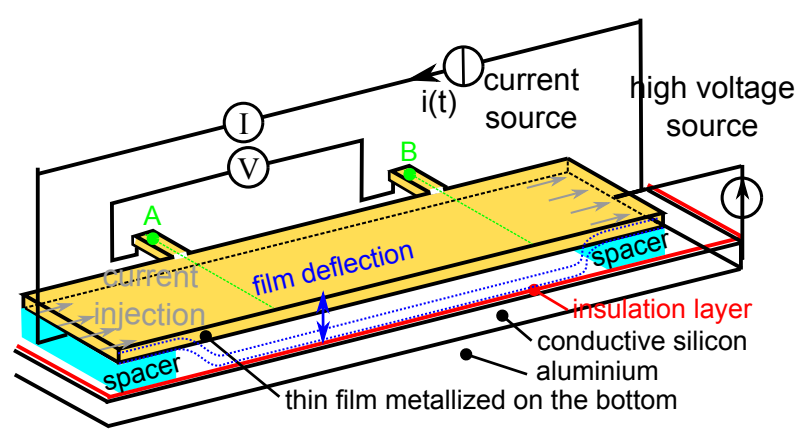

FIG. 3. Detailed isometric view of our experiment with the voltage, the current measurement and the high voltage source. At $0 \mathrm{~V}$ the film is at rest (black lines), and at $300 \mathrm{~V}$ the film is pulled to the lower part.

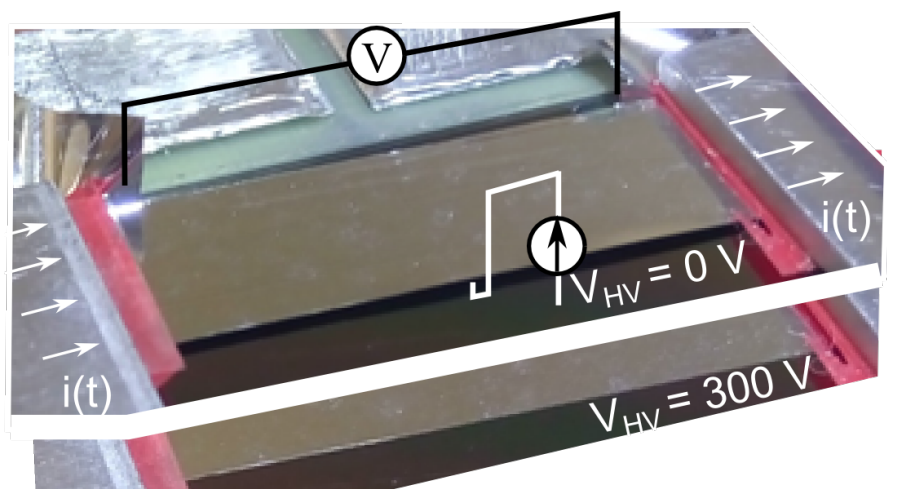

FIG. 4. On the top, the film is in "off" state, the voltage is not applied $V_{H T}=0$ and the film is suspended. On the bottom, the film is in the "on" state, the voltage is applied $V_{H T}=300 \mathrm{~V}$ and the film is in contact. A voltmeter used to measure the resistance is set up between the point $\mathrm{A}$ and $\mathrm{B}$.

deposited by spin-coating that can withstand to $400 \mathrm{kV} \mathrm{mm}^{-1}$. After depositing the polymer film a great care has been taken to maintain a good surface/interface contact between the film and the polished surface of the wafer even though the system has not been assembled in a clean-room environment. The size of the active part of the system and especially its thickness are at the boundary of Micro-Electro-Mechanical Systems (MEMS) based technologies where electrostatic actuation is commonly used as a transduction mechanism. Indeed, electrostaticdriven MEMS devices have already been well studied ${ }^{14}$. As our main focus is on the study of the thermal characterization of the switch, our electrostatic actuator time response is not optimized; the switching time is around several seconds. The wafer is pressed on bulk aluminum $(12 \mathrm{~cm} \times 12 \mathrm{~cm})$ with an unknown air gap in-between due to the roughness of the surface.

The current $I$ going through the film and the voltage $V_{A / B}$, are measured as shown in the Fig. 3. The temperature is estimated through the measured resistance. When metallic films are thin enough (below $\sim 1 \mu \mathrm{m}$ ) the temperature coefficient of the resistance ${ }^{15,16}$ (TCR) de- 
creases as a function of thickness. In our case, bulk $\mathrm{Al}$ TCR is commonly around $4.3 \times 10^{-3} \mathrm{~K}^{-1}$, and remains constant $^{15}$ at least down to $180 \mathrm{~nm}$. As we estimate the metallization to be around $10 \mathrm{~nm}$, we checked the TCR using an experimental setup, described in detail elsewhere $^{17}$, based on the four-probe method used under high vacuum $\left(10^{-6}\right.$ mbar $)$ with an in-situ heat control device. We find a linear dependence with a TCR of $(1.01 \pm 0.01) \times 10^{-3} \mathrm{~K}^{-1}$. The measurement gives the power dissipated by Joule effect and the temperature of the metallized film. Because the size of the working area $(1 \mathrm{~cm} \times 2.9 \mathrm{~cm})$ is larger than the the thickness of the system and the current density is homogeneous across the film (i.e the "strip" used for the voltage measurement has a negligible impact on it), our study is restricted to the out of plane heat flux, thus we can use a 1D diffusion model. This 1D model is based on a finite volume method for the spatial discretization and used Runge-Kutta timestepping schemes. The evaluation of the thermal conductance $k$ requires the knowledge of the thermal flux that goes through the interface, the temperature of the metallization and the one of the the insulation layer, the latter being rather difficult to measure. Because the interface thermal conductances (i.e. film/PI and wafer/Al) are not directly accessible, they are extrapolated using a 1D thermal diffusion model through the different thermal responses of the system and the knowledge of thermal properties of the different materials in the stack, of their thicknesses and of the power injected.

Performing synchronous detection (i.e successive capture averaging) over a stationary thermal behavior we improved the signal to noise ratio (i.e. an average of 60 periods). Moreover, we removed the DC component to adjust the the low frequency drift.

The current intensity passing through the metallization follows a step pattern: $I=I_{\min }$ for $t \in[0,1.5] \cup[2.5,5]$ and $I=I_{\max }$ for $t \in[1.5,2.5]$. In the off state, $I_{\max }$ goes down to $200 \mathrm{~mA}$, whereas in the on one it goes up to $300 \mathrm{~mA}$. Roughly, this corresponds to a current density of $3000 \mathrm{~A} \mathrm{~mm}^{-2}$ through the film. In the on state, the current is increased in order to have significant temperature change (around $1 \mathrm{~K}$ ) despite the improvement of the thermal conductance. Figure 5 shows an example of temperature variation for different currents in on state. We use a periodic current pulse rather than a sinusoidal one (3-omega method) to maximize the high frequency thermal response where the effect of the interface is predominant and to limit the average temperature increase at the extremities of the film which are always suspended. As the injected power shapes are linked by a linear transformation which can be used within the 1D diffusion equation, the temperature responses follow the very same linear transformation (i.e. the temperatures measured are related to each other). Applying a normalization transformation for different current values, all measurements rescale towards the same curve as shown in Fig. 5.

In the off state, the model allows to single-out the joint

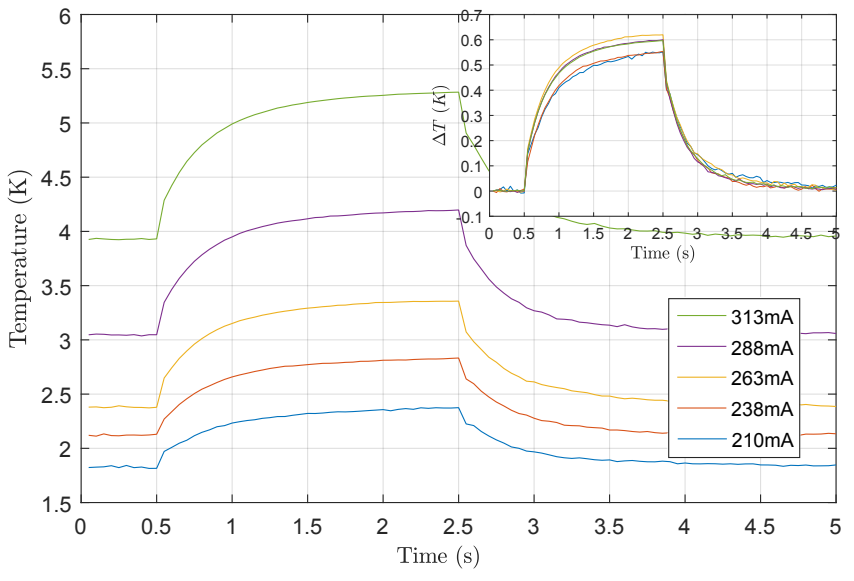

FIG. 5. Temperature measurement in the on state for different current pulses where $I_{\min }=52 \mathrm{~mA}$ and $I_{\max }$ is indicated in the legend. The inset shows the temperature variation for different current pulses normalized with the reference $I_{\max }=210 \mathrm{~mA}$

thermal responses associated with the film thermal capacity and with two different thermal resistances in parallel. One is associated with exchanges with ambient air, the other with the air gap between the film and the wafer. The estimation of heat exchange coefficient and of the air gap are difficult to disentangle. However, assuming an air heat transfer coefficient of $h=8 \mathrm{~W} \mathrm{~K}^{-1} \mathrm{~m}^{-2}$ we estimate an air gap thickness of $975 \mu \mathrm{m}\left(25 \mathrm{~W} \mathrm{~K}^{-1} \mathrm{~m}^{-2}\right)$, which is in agreement with the actual set-up.

In the case of the on state, as the conductance between the film and the wafer drastically increases, the heat diffusion between the different material layers plays an key role. Table I shows the thermal characteristic of the layers. As the amount of air trapped in-between the film/PI and the wafer/Al interface is hardly knowable, we model it with an equivalent air-gap adjusted to fit the measured response.

\begin{tabular}{|c|c|c|c|c|}
\hline Material & Length $(\mu \mathrm{m})$ & $C\left(\mathrm{~mJ} \mathrm{~K}^{-1}\right)$ & $K\left(\mu \mathrm{K} \mathrm{W}^{-1} \mathrm{~m}^{-2}\right)$ & $\tau(\mathrm{ms})$ \\
\hline film & 8 & 8.2 & 53 & 0.8 \\
\hline Air (On) & $\approx 1$ & $\approx 0$ & 40000 & $\approx 0$ \\
\hline Air (Off) & $\approx 1000$ & $\approx 0$ & 40 & $\approx 0$ \\
\hline PI & 5 & 4.1 & 25 & 0.2 \\
\hline Si & 450 & 475 & 3.4 & 2.8 \\
\hline Air & $\approx 1$ & $\approx 0$ & 40 & $\approx 0$ \\
\hline Al & $3.10^{4}$ & $41.10^{3}$ & 125 & $4.10^{3}$ \\
\hline
\end{tabular}

TABLE I. Length and thermal properties of layers with the volumic thermal capacity, the thermal conductance and the characteristic diffusion time, metallization is too small to affect the thermal behavior

Using the characteristics of the layers and the 1D diffusion model, the temperature response of the metallization is deduced. The response shows three separate time scales: a fast one, around $10 \mathrm{~ms}$, a medium one around $1 \mathrm{~s}$ and a slow one of several seconds. These different time scales are related to the thermal diffusion through 
the different layers.

The slow response time (the small slope at the end of pulse in Fig. 6) corresponds to a state where the temperature has become homogeneous across the system and it shows the dissipation from the system as a whole to the environment. This becomes apparent when we see that all the curves have the same asymptotic behavior. The intermediate response time $(\sim 1 \mathrm{~s})$ strongly depends on the thermal conductance of the two air gap interfaces (Film/PI and Wafer/Al) as well as on the thermal capacity of the wafer. At this scale when current is switched to $I_{\min }$, all the curves converge to the same tail because the heat exchange is limited by the Wafer/Al air gap. The fast response time reveals the effect of the thermal conductance of the studied interfaces (i.e Film/PI) as shown by the simulation for different air gaps in the Fig. 7 . A careful fitting of this initial step provides a reliable estimation of the researched thermal conductance.

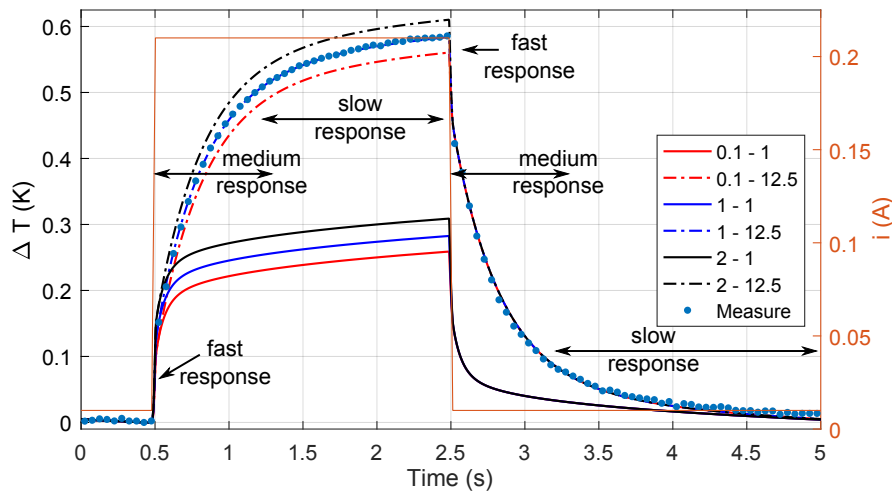

FIG. 6. Simulation of the temperature variation for different air gap layers (thicknesses in $\mu \mathrm{m}$ in the legend). The first value indicates the air gap of the Film/PI interface and the second indicates the air gap of the Wafer/Al interface

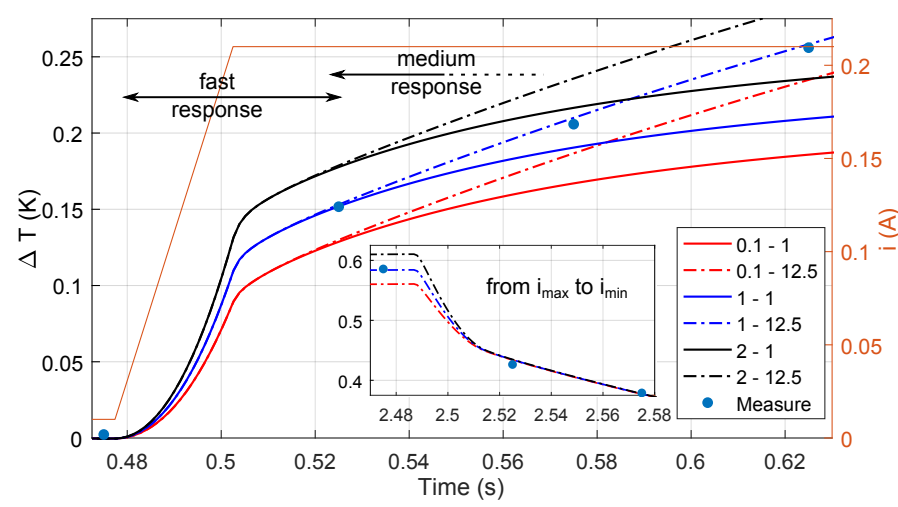

FIG. 7. Zoom on the fast response time and dependencies of the Film/PI air gap thickness as well as the independence of the Wafer/Al air gap. In the inset, the temperature variation when the pulse is descending.

The thermal conductance of the Film/PI interface is estimated around $25 \mathrm{~kW} \mathrm{~K}^{-1} \mathrm{~m}^{-2}$ (1 $\mathrm{\mu m}$ air gap). Con- sidering the surface flatness and roughness of the PI deposed on a polished optical surface, the value of $1 \mu \mathrm{m}$ seems unexpectedly high. However air and dust were presumably trapped in the interface during the collapse of the film and this can explain the large value and some discrepancy between different sets of measurement. This means that further improvements can be achieved by assembling the system in a clean room. It is worth noting that whereas thermal conductance foreseen in Ref. ${ }^{12}$ comes from theoretical calculations our result stems from experiments and therefore it represents a extremely promising milestone for TSs optimization. Comparison to existing thermal switches ${ }^{4,10}$ shows a ratio 10 times higher with a thermal conductance ratio $\eta=10^{3}$. In the following section, we will show that our results provide a potential technological breakthrough for solid state cooling.

A caloric thin film integrated between two electrostatically actuated thermal switches is a good candidate for a high power density cooling device showing efficient thermal exchange due to a high surface-to-thickness ratio. Moreover, such a system can be easily stacked, thus allowing an increase of the temperature span. Power density of a cooling device is estimated in the framework of finite time thermodynamics with a Carnot endoreversible cycle where at maximum efficiency ${ }^{2}$, the power density $P_{\text {cold }}$ and the relative efficiency $\epsilon_{\text {rel }}$ are approximated by

$$
\begin{gathered}
P_{\text {cold }} \approx \frac{\Delta T_{\text {adia }}}{2} \frac{k_{\text {on }}}{d} \frac{\sqrt{\eta}-1}{\sqrt{\eta}+\eta} \\
\epsilon_{\text {rel }} \approx\left(\frac{\sqrt{\eta}-1}{\sqrt{\eta}+1}\right)^{2} \approx\left(\frac{T_{\text {hot }}-T_{\text {cold }}}{\Delta T_{\text {adia }}}\right)^{2}
\end{gathered}
$$

where $\Delta T_{\text {adia }}$ is the adiabatic temperature change associated to the caloric material, $T_{\text {hot }}$ and $T_{\text {cold }}$ the temperature of the hot and of the cold reservoirs and $d$ is the active material film thickness. Different endoreversible cycles are possible, among those cycles we choose the cycle working at maximum efficiency. Using $\Delta T_{\text {adia }}$ of the electrocaloric $\mathrm{P}(\mathrm{VDF}-\mathrm{TrFE}-\mathrm{CFE})^{1,13}$ namely $10 \mathrm{~K}$, $d=10 \mu \mathrm{m}$, the $k_{\text {on }}$ and the $\eta$ of our switch and considering a numerical computation of the efficiency similar to the one $\mathrm{in}^{2}$, the relative efficiency is $85.7 \%$ of the Carnot efficiency. The cycle works with a temperature difference of the reservoir $T_{\text {hot }}-T_{\text {cold }}$ of $9.3 \mathrm{~K}$ which produce a cooling power of $0.410 \mathrm{~W} \mathrm{~mm}^{-3}\left(228 \mathrm{~W} \mathrm{~g}^{-1}\right)$. The result represents an upper bound for the efficiency because the only entropy production taken into account is the one related to the heat flow whereas we neglect any other source of entropy production such as Ohmic heating, hysteresis... Assuming a caloric material that exchanges $10 \%$ of its entropy change ${ }^{1}$ at each cycle $30 \mathrm{~mJ} \mathrm{~mm}^{-3}\left(17 \mathrm{~J} \mathrm{~g}^{-1}\right)$, we obtain a working frequency around $100 \mathrm{~Hz}$. The increase of the frequency requires switching the film in less than $1 \mathrm{~ms}$ and this has not been demonstrated so far. This shows that the $k_{\text {on }}$ value we obtained for our thermal switch represents a major opportunity to develop a 
next generation of more efficient solid state cooling devices as recently demonstrated $\mathrm{in}^{13}$. Actually the device presented in ${ }^{13}$ uses an electrostatic switch very similar to the one discussed here. However ${ }^{13}$ is focused on the performance of the whole cooling device. On the contrary we present a detailed TS characterization, without the active substance, with a particular focus on its time response. In this way our data can be used to model the thermodynamic cycle of the device using a generic caloric material. Indeed, decoupling the thermodynamic of the active part from that of thermal exchange is a key feature to properly model thermal engines ${ }^{18}$. Finally, using our data, we are able to show that with a wise thermal management the system presented in ${ }^{13}$ can be improved leading to a power density up to $228 \mathrm{~W} \mathrm{~g}^{-1}$ with a relative efficiency of $85 \%$ if the switching time could be mastered lower than $1 \mathrm{~ms}$. This work has benefited from the financial support of the LabeX LaSIPS (ANR-10LABX-0040-LaSIPS) managed by the French National Research Agency under the "Investissements d'avenir" program (ANR-11-IDEX-0003-02).

${ }^{1}$ X. Moya, S. Kar-Narayan, and N. D. Mathur, "Caloric materials near ferroic phase transitions," Nat Mater 13, 439-450 (2014).

${ }^{2}$ R. I. Epstein and K. J. Malloy, "Electrocaloric devices based on thin-film heat switches," Journal of Applied Physics 106, 064509 (2009).

${ }^{3}$ M. Almanza, A. Kedous-Lebouc, J.-P. Yonnet, U. Legait, and J. Roudaut, "Magnetic refrigeration: recent developments and alternative configurations*," Eur. Phys. J. Appl. Phys. 71, 10903 (2015).

${ }^{4}$ T. Kato, T. Nagahara, Y. Agari, and M. Ochi, "Relation between thermal conductivity and molecular alignment direction of freestanding film aligned with rubbing method," Journal of Polymer Science Part B: Polymer Physics, 43, 3591 - 3599 (2005).

${ }^{5}$ H. Tian, D. Xie, Y. Yang, T.-L. Ren, G. Zhang, Y.-F. Wang, C.-J. Zhou, P.-G. Peng, L.-G. Wang, and L.-T. Liu, "A novel solid-state thermal rectifier based on reduced graphene oxide," 2, 10.1038/srep00523.

${ }^{6}$ D. Sawaki, W. Kobayashi, Y. Moritomo, and I. Terasaki, "Ther- mal rectification in bulk materials with asymmetric shape," Applied Physics Letters 98, 081915.

${ }^{7}$ S. Li, X. Ding, J. Ren, X. Moya, J. Li, J. Sun, and E. K. H. Salje, "Strain-controlled thermal conductivity in ferroic twinned films," 4, 10.1038/srep06375.

${ }^{8}$ J. B. Boreyko, Y. Zhao, and C.-H. Chen, "Planar jumpingdrop thermal diodes," Applied Physics Letters 99, 234105 (2011), http://dx.doi.org/10.1063/1.3666818.

${ }^{9}$ A. R. McLanahan, C. D. Richards, and R. F. Richards, "A dielectric liquid contact thermal switch with electrowetting actuation," Journal of Micromechanics and Microengineering 21, 104009 (2011).

${ }^{10}$ J. Cho, T. Wiser, C. Richards, D. Bahr, and R. Richards, "Fabrication and characterization of a thermal switch," Sensors and Actuators A: Physical 133, 55-63 (2007).

${ }^{11}$ Y. Wang, D. E. Schwartz, S. J. Smullin, Q. Wang, and M. J. Sheridan, "Silicon Heat Switches for Electrocaloric Cooling," Journal of Microelectromechanical Systems 26, 580-587 (2017).

${ }^{12}$ H. Keum, M. Seong, S. Sinha, and S. Kim, "Electrostatically driven collapsible $\mathrm{Au}$ thin films assembled using transfer printing for thermal switching," Applied Physics Letters 100, 211904 (2012).

${ }^{13}$ R. Ma, Z. Zhang, K. Tong, D. Huber, R. Kornbluh, Y. S. Ju, and Q. Pei, "Highly efficient electrocaloric cooling with electrostatic actuation," Science 357, 1130-1134 (2017).

${ }^{14}$ W.-C. Chuang, H.-L. Lee, P.-Z. Chang, and Y.-C. Hu, "Review on the Modeling of Electrostatic MEMS," Sensors 10, 6149-6171 (2010).

${ }^{15}$ F. Aviles, O. Ceh, and A. Oliva, "Physical properties of au and al thin films measured by resistive heating," Surface Review and Letters 12, 101-106 (2005).

${ }^{16}$ S. E. Gustafsson, E. Karawacki, and M. N. Khan, "Simultaneous measurement of resistivity and temperature coefficient of resistivity of metallic thin films with the transient hot-strip method," Thin Solid Films 92, 287-294 (1982).

${ }^{17}$ S. Lemettre, S. Hammami, A. Bosseboeuf, P. Coste, and J. Moulin, "In-situ electrical characterization of co-evaporated zr-v, zr-ti and zr-co thin getter films during thermal activation," in Design, Test, Integration and Packaging of MEMS/MOEMS (DTIP), 2017 Symposium on (IEEE, 2017) pp. 1-4.

${ }^{18}$ B. Andresen, R. S. Berry, M. J. Ondrechen, and P. Salamon, "Thermodynamics for processes in finite time," Accounts of Chemical Research 17, 266-271 (1984). 